Surg Radiol Anat (1997) 19: 119-121

(C) Springer-Verlag France 1997

Technical note

\title{
An in vitro anatomic model of the human cerebral arteries with saccular arterial aneurysms
}

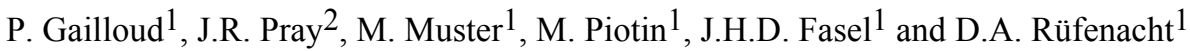

${ }^{1}$ Division of Diagnostic and Interventional Radiology, Department of Morphology, and Clinical Anatomy Research Group (GRAC), University of Geneva, Switzerland

${ }^{2}$ Boston Scientific Corporation, Natick, MA, USA

Received November 5, 1996 / Accepted in final form January, 16, 1997

Key words: Vascular model Methodology Reproducibility

Correspondence to: P. Gailloud, Division of Diagnostic and Interventional Radiology, H.U.G., CH-1211 Geneva 14, Switzerland

\begin{abstract}
An in vitro model of the main human cerebral arteries with or without saccular arterial aneurysms is presented. A cast of the cerebral arteries was obtained in a human specimen. Three aneurysms were simulated and added to the cast. Wax copies of the cast were produced, and embedded with liquid resin solidifying into solid blocks. After evacuation of the wax, a model consisting of a hollow reproduction of the cast within the resin block was obtained. The model is reproducible and anatomically accurate. Since it is transparent to visible light, and compatible with x-ray, magnetic resonance and transcranial doppler techniques, it should prove useful for a wide range of haemodynamic and radiologic investigations. The reported technique may be adapted to any structure with a hollow configuration, allowing for the preparation of arterial and venous models from other vascular areas, as well as models from other anatomic systems, such as the biliary or urinary tracts.
\end{abstract}

The development of reliable models of the normal and pathologic cerebral circulation is mandatory to allow in vitro research activities such as haemodynamic studies or development and evaluation of new vascular imaging and endovascular therapeutical methods. A reproducible and anatomically accurate model of the human cerebral arteries, with or without the adjunction of saccular arterial aneurysms, is described.

\section{Material and methods}

A corrosion cast of the aortic arch and the cervicocranial arteries was obtained by injecting a mixture of methylmethacrylate (Beracryl, Troller, Switzerland) and baryum sulfate powder (HD 200 plus, Lafayette, USA) into the arterial tree of a non-fixed human cadaver $(72 \mathrm{~F})$ under fluoroscopic control. The mixture was injected after flushing of the arterial tree and injection material with a $5 \%$ glucose solution. Once injected, the specimen was immersed in a $15 \%$ solution of potassium hydroxide $(\mathrm{KOH})$ at $40^{\circ} \mathrm{C}$ until complete elimination of the surrounding soft and bony tissues was achieved. The corrosion cast was subsequently simplified by ablating small branches, leaving a cleaned cast consisting of the main cervicocranial arteries. The cast chosen for the model presented and evaluated in this paper was a right internal carotid artery showing typical anatomic variants known to provide conditions predisposing to the development of certain cerebral aneurysms. The casts of three aneurysm cavities of different shape and size were simulated using methylmethacrylate and added to the corrosion cast at typical implantation sites, such as the origin of a posterior communicating artery $(\mathrm{PCoA})$, at the anterior communicating artery $(\mathrm{ACo} A)$ in the presence of an ipsilateral dominant A1 segment, and at the middle cerebral artery (MCA) bifurcation.

Using dentistry prosthetic techniques, 300 wax copies of the arterial cast with the three aneurysms were then obtained (Fig. 1). Zero to two aneurysms were ablated from the different wax copies in order to produce specific individual models with one to three aneurysms. The wax copies were afterwards embedded in a clear liquid resin (EP 4101, Eager Plastics, Chicago, USA) solidifying into solid transparent blocks. Holes were drilled in the blocks reaching the wax at different points, allowing for subsequent evacuation of the wax by heating. A model consisting in a hollow reproduction of the arterial cast within a transparent block was thus finally obtained (Fig. 2). 


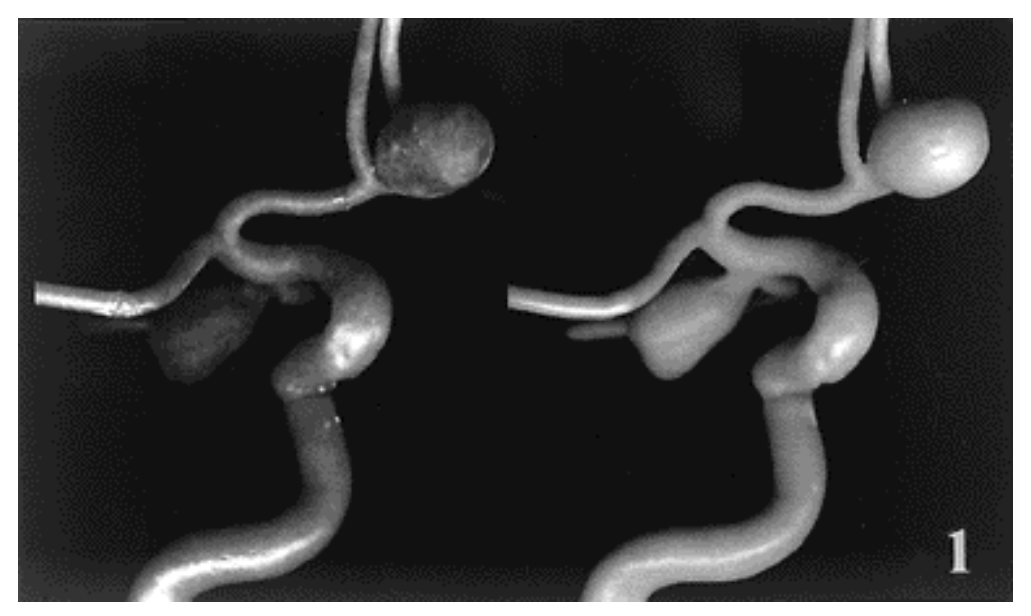

Fig. 1

The right internal carotid artery corrosion cast used for the preparation of the models is showed on the left side. Note the simulated ACoA and PCoA aneurysms (the MCA aneurysm is not visible on this picture). Three hundred wax copies of this initial cast were produced (right side), with a moulding technique allowing for excellent reroducibility of the cast configuration

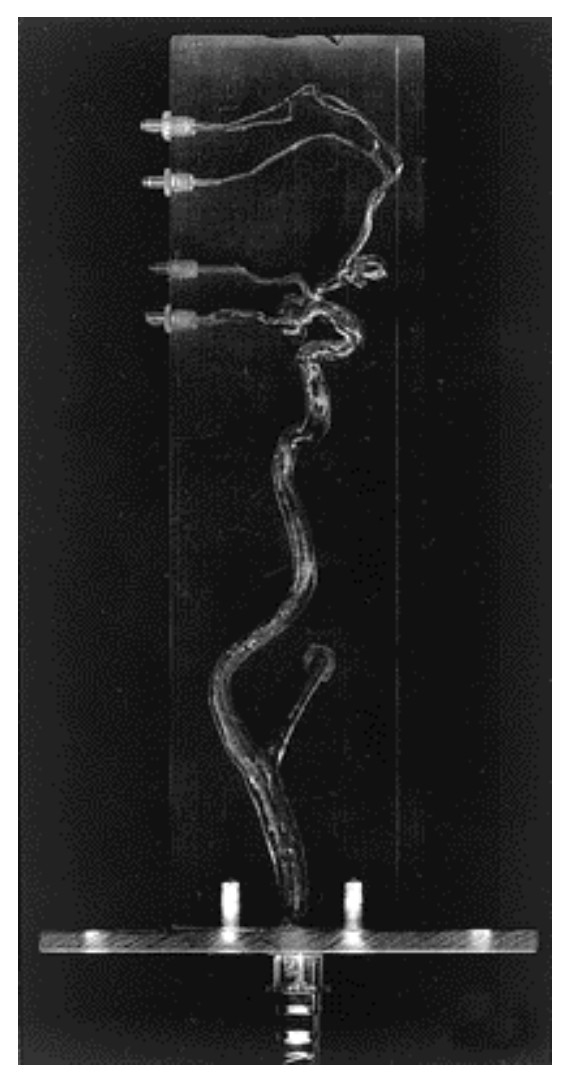

Fig. 2

The final model consists of a hollow reproduction of the initial arterial tree within a solid and transparent block. Note the presence of connectors allowing for the branching of a pump delivering a pulsatile flow inside the model lumen

In order to assess possible variations in calibres induced by the procedure used in the creation of the models, the full process was reproduced with 6 methylmethacrylate cylinders of different sizes $(3,4,5,10,15$ and $25 \mathrm{~mm})$. Two resin models were obtained from each cylinder. Digital x-ray images were taken at each step of the procedure and calibres were evaluated using a built-in measurement software (Integris, Philips, Netherland). X-ray absorption coefficients of the models were estimated using computerized tomography (Hounsfield units measurements). Compatibility of the models with 1.5 Tesla magnetic resonance (MR) and transcranial doppler (TCD) devices was finally evaluated.

\section{Results and discussion}

Reproducibility The in vitro models were derived from vascular casts by application of a molding procedure, as formerly proposed by Liepsch and Zimmer [3] and Kerber et al [2]. Our methodology has the capacity to provide an unlimited number of identical wax copies from a single initial vascular cast. The measurements performed on simple cylinders (Table 1) showed that the variation in calibre induced by the procedure used for the preparation of the resin blocks could be considered negligible. The conditions for the production of highly reproducible vascular models, as for example recquired for comparative testing, are thus provided. 


\begin{tabular}{rcc}
\hline Real size & $M$ & $R$ \\
\hline $25 \mathrm{~mm}$ & 100 & $97.7 \pm 1.4$ \\
$15 \mathrm{~mm}$ & 100 & $99.4 \pm 1.3$ \\
$10 \mathrm{~mm}$ & 100 & $101.9 \pm 1.7$ \\
$5 \mathrm{~mm}$ & 100 & $101.6 \pm 2.2$ \\
$4 \mathrm{~mm}$ & 100 & $103.1 \pm 2.8$ \\
$3 \mathrm{~mm}$ & 100 & $99.7 \pm 4.2$ \\
\hline
\end{tabular}

\section{Table 1}

Comparison of calibres between methylmethacrylate cylinders $(\mathrm{M})$ and resin models $(\mathrm{R})$. Since variations in calibres rather than absolute values were to be evaluated, $M$ was used as reference for the calibration of the software measurement tool, being given the arbitrary value of 100 . The values reported for R (average value of 12 measurements obtained from 2 resin models) may thus be read directly as a percentage of the $\mathrm{M}$ value

\section{Morphological accuracy}

Initial injection of methylmethacrylate performed under fluoroscopy provided a continuous visual control of the procedure, allowing for the injection to be interrupted as soon as the relevant arteries were filled. Potential alteration of the arterial morphology by excess injection pressure of the casting material was avoided [1]. Obtaining a corrosion cast from a human specimen provided models matching the complexity of the human cerebral vascular tree.

With our technique, secondary modifications of the arterial configuration may be produced in two different manners. The modifications may be performed on the corrosion cast itself, and thus be precisely reproduced on each wax copy which is thereafter obtained (i.e. the aneurysms added to the present model). But the modifications may also be performed directly on the wax copies, allowing for the production of models with individual characteristics (i.e. aneurysmal size and shape modifications in the present model). Specific alterations, as for example changing of the branching angle of a vessel or addition of a pathologic characteristic like a stenosis, are thus easily produced on models which are otherwise perfectly comparable. The influence of these specific alterations on the haemodynamic characteristics of the arterial tree may then be evaluated selectively.

The arterial cast chosen for the model preparation showed anatomic characteristics known to be related to the development of cerebral aneurysms, such as a large (fetal-type) PCoA and a markedly dominant A1 segment of the ACA with a large ACoA [4]. The simulated aneurysms were implanted at classic locations of aneurysmal development, corresponding to approximately two thirds of the aneurysms occuring in the anterior cerebral circulation [4].

\section{Physical properties}

The material used for the model (EP 4101, Eager Plastics, Chicago, USA) is resistant to a wide range of chemical products and solvants. It is transparent to visible light, moderatly radioopaque (ca 107 Hounsfield Units) (Table 2), and produces neither artifacts nor a signal when tested in a MR device at 1.5 Tesla. Though the model was not primarely intended to be used with ultrasound techniques, a signal of good quality was obtained using a TCD device while a blood-equivalent fluid was circulating through the model. The compatibility of the model with different radiologic and non-radiologic imaging modalities, and its potential utilization as an experimental environment for endovascular techniques simulations are presently under evaluation.

\begin{tabular}{lc}
\hline & Coeffident (in HU) \\
\hline Air & -1000 \\
Water & 0 \\
Model & $107 \pm 3$ \\
Bone & 1000 \\
\hline
\end{tabular}

\section{Table 2}

X-ray absorption coefficients in Hounsfield units (HU). The model value is the average value of 32 measurements made on 4 different models

\section{Further perspectives}

The reported methodology is adaptable to any structure presenting a hollow configuration, allowing for the preparation of arterial and venous models from other vascular areas, as well as models from other anatomic systems, such as the biliary or urinary tracts. Moreover, other types of embedding material, such as silicone, may be applied to the wax copies in order to obtain models with different physical characteristics.

\section{Conclusion}

An anatomic model of the human cerebral arteries is presented. This model is highly reproducible and matches the complexity of the human arterial tree. Saccular aneurysms secondarly implanted on the arterial cast are shown as an example of pathologic conditions which may be simulated on this model. The model is produced at low material costs and, since it is transparent to visible light and compatible with $\mathrm{x}$-ray, MR and TCD techniques, it should prove useful for a wide range of haemodynamic and 
radiologic investigations, as well as for teaching purposes.

Ackowledgements. This work was supported by the Swiss National Science Foundation (SNSF Project \#32-42529.94). The authors would also like to express their thanks to L. Auer and W. Weber, MD hc, for their valuable contribution to the present work.

\section{References}

1. Gailloud P, Muster M, Besse S, Rüfenacht DA, Fasel JHD (1995) Apport de l'angiographie digitalisée postmortem à la recherche en anatomie vasculaire. J Radiol 76: 800-801

2. Kerber CW, Heilman CB, Zanetti PH (1989) Transparent elastic arterial model I: a brief technical note. Biorheology 26: 10411049

3. Liepsch D, Zimmer R (1978) Verfahren zur Herstellung maßstabsgetreuer starrer und naturgetreuer elastischer menschlicher Arterienmodelle. Biomed Techn 23: 227-230

4. Redekop G, Ferguson G (1995) Intracranial Aneurysms. In: Carter LP, Spetzler RF, Hamilton MG (eds) Neurovascular Surgery. McGraw-Hill, New York, pp 625-648

Back to the SRA-EE Home Page

Last change: June 9, 1997

helpdesk.link@springer.de

(C) 1997 by Springer-Verlag France 